\title{
ANTERIOR OSTEOPHYTE IDENTIFICATION IN CERVICAL VERTEBRAE
}

\author{
Ms. A. T. Chougale ${ }^{1}$ and Mr. N. B. Sambre. ${ }^{2}$ \\ ${ }^{1}$ Department of Electronics \& Telecommunication Engineering, K.I.T.'s college of \\ Engineering, Kolhapur, Maharashtra. \\ ashwinishinde2112@gmail.com \\ ${ }^{2}$ Department of Electronics \& Telecommunication Engineering, K.I.T.'s college of \\ Engineering, Kolhapur, Maharashtra. \\ sambrenitin@yahoo.com
}

\begin{abstract}
Radiologist always examines X-ray to determine abnormal changes in cervical, lumbar \& thoracic vertebrae. Osteophyte (bony growth) may appear at the corners of vertebrae so that vertebral shape becomes abnormal. This paper presents the idea from Image processing techniques such as customised Hough transform which will be used for segmentation which should be independent of rotation, scale, noise \& shape. This segmented image will be then used for computing size invariant, convex hull based features to differentiate normal cervical vertebrae from cervical vertebrae containing anterior osteophyte. This approach effectively finds anterior osteophytes in cervical vertebrae.
\end{abstract}

\section{KEYWORDS}

Osteophytes, customised Hough transform, convex hull, segmentation.

\section{INTRODUCTION}

Radiographs of the spine provide a practical approach for detecting and assessing vertebral abnormalities that may be related to osteoarthritis or osteoporosis. The presence of bony growths ('Osteophyte') on vertebra corners, disc space narrowing are all features commonly evaluated visually from radiographs.

The presence of osteophytes is significant because it may be related to degeneration in the attachment of the outer annular fibers of the disc to the vertebral endplate. This degeneration may allow the vertebra to slip to the anterior or to both the anterior and the side [12]. Vertebra distortion based on anterior osteophytes results in a deviation from the characteristic rectangular vertebral shape, which tends to be convex. This proposed work focuses on vertebral distortion along the anterior boundary as an indicator of Osteophyte. Fig. 1 presents three examples of anterior osteophytes in vertebrae with osteophytes in the upper left-hand corner (a), osteophytes in the lower left-hand corner (b) and osteophytes in both left-hand corners (c). Fig. 3 shows a normal vertebra (left-hand side is the anterior side). 

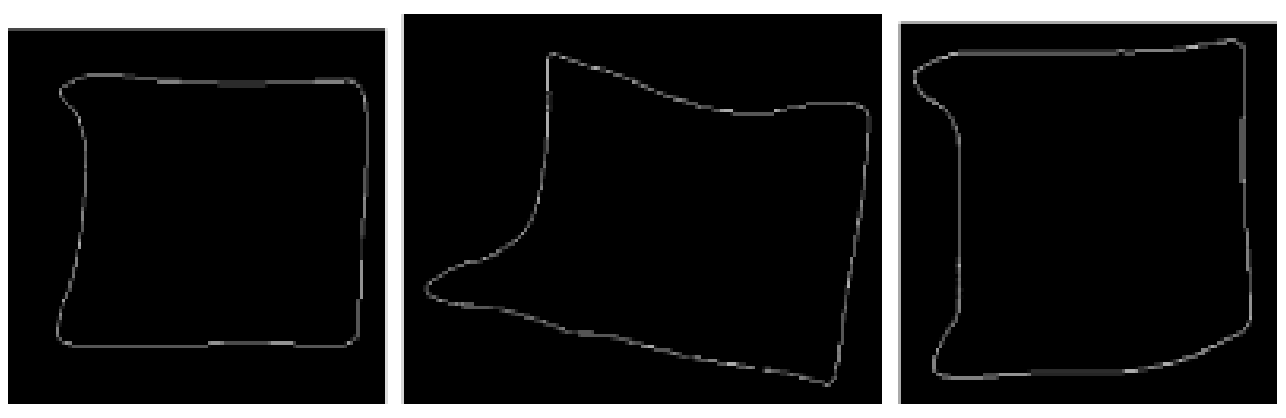

Figure.1. Three examples of anterior osteophytes vertebrae with osteophytes The left-hand side is the anterior side of the vertebra shown in upper left-hand corner (a), osteophytes in the lower left-hand corner (b), and osteophytes in both left-hand corners (c)

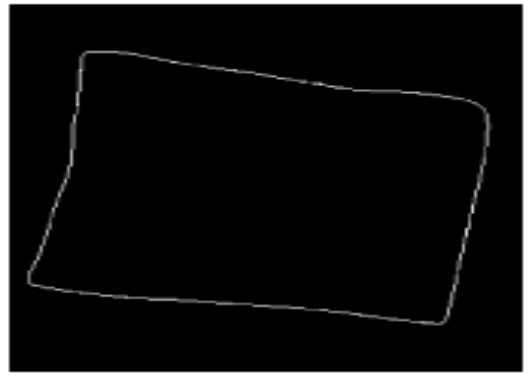

Fig.2. Example for normal vertebra without anterior osteophytes

GHT uses an edge image to correlate points in a previously defined template to those in the target image using local gradient information. If a good edge image is not available, too few votes will be correlated to the template [1]. GHT assume that a clear edge image, from which gradient information can be obtained. Since x-ray images are not clear edge image, an edge detection process is used. The proposed process performs Gaussian blurring on a copy of the original image and the result is subtracted from the original. From the resulting edge image, extraction of the gradient information will be performed using optimum gradient operators.

In prior research, image processing techniques were investigated to compute features along the anterior boundary of cervical spine vertebrae for differentiating normal vertebrae from vertebrae with abnormal osteophytes [13]. The features examined were radius-of-curvature-and grayscale gradient-based features computed along the anterior boundary of cervical spine vertebrae. The radius of curvature-based features highlights the relative constriction along the anterior boundary between normal and abnormal vertebrae. The grayscale border gradient features examine the grayscale contrast difference between the vertebral interior and exterior along the boundary. The primary difficulty with the radius-of-curvature features was that they were not adaptive to vertebral size. In this research, size-invariant convex hull features are introduced to detect anterior osteophytes in cervical spine vertebra. Size-invariant features are investigated in order to facilitate the direct comparison of vertebrae between radiographs.

In this proposed work, size-invariant convex hull features will be used to detect anterior osteophytes in cervical spine vertebra. Size-invariant features will investigate for the direct comparison of vertebrae between radiographs. The convex hull of a data set of points is the 
smallest convex set of points that includes the data set of points. The convex hull will determined from a vertebra's boundary points in order to compute features characterizing the vertebra's shape. Examining Figs. 1 and 2, a normal vertebra generally has rectangular shape and tends to be convex. Thus, the convex hull for a cervical spine without anterior osteophytes is anticipated to be similar to its original shape on the anterior side. Inspecting Fig. 1, the protrusion region along the anterior side of the vertebra contains osteophytes, making the vertebra shape concave. Four size-invariant features are determined based on comparing a filled vertebra with its filled convex hull.

A standard multi-layer perceptron (MLP) will be used to evaluate the features for osteophytes discrimination on a substantial lumbar vertebrae data set [2].

\section{ALGORITHM FOR OSTEOPHYTES IDENTIFICATION IN CERVICAL VERTEBRAE}

In this proposed work, image analysis techniques are investigated for detecting abnormal (i.e. having osteophytes) cervical spine vertebrae. For individual vertebra analysis automatic vertebral segmentation will be performed. The steps for automatic vertebra segmentation, vertebral feature calculations, and vertebral discrimination are presented in following sections.

\subsection{VERTEBRAL BOUNDARY DETERMINATION:}

The customized Hough Transform will be used to captures shape variability and exploits shape information embedded in the Hough domain to overcome noise. The following subsections describe the different processing steps involved in the CHT [10].

\subsubsection{Extraction of gradient information:}

Here unsharp masking is proposed to obtain edge image. Mathematically the resulting image after unsharp masking can be represented as:

$$
\mathrm{Fu}(\mathrm{x}, \mathrm{y})=\mathrm{f}(\mathrm{x}, \mathrm{y})-\mathrm{fb}(\mathrm{x}, \mathrm{y})
$$

Where $f(x, y)$ represents the original image [Figure.3 (a)], $f b(x, y)$ represents the Gaussian blurred image [Figure.3 (b)], and fu(x,y) represents the unsharp masked image [Figure.3(c)]. Here subtraction of a blurred image from the original will provide only abrupt local variations in edges and eliminate an unnecessary information (background). An averaging filter is used to reduce the unwanted high frequency components. Then the resulting edge image is binaries. Finally gradient operators are passed to obtain edge image. The Sobel and Prewitt operators can be used to estimate the gradient information but there is inconsistency between the discrete and continuous gradient information. This can be minimized using the gradient descent method [6]. The optimum $3 \times 3$ gradient operator is given as:

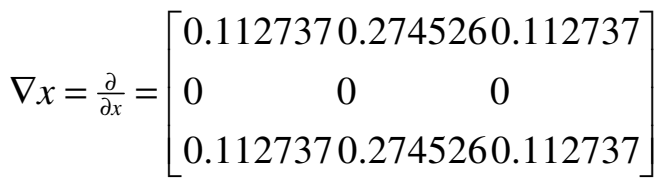

$$
\begin{aligned}
& \nabla y=\frac{\partial}{\partial y}=\left[\begin{array}{lll}
0.112737 & 0 & 0.112737 \\
0.274526 & 0 & 0.274526 \\
0.112737 & 0 & 0.112737
\end{array}\right]
\end{aligned}
$$




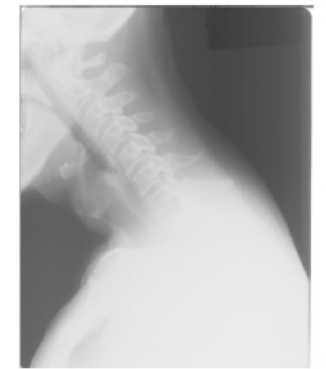

(a)

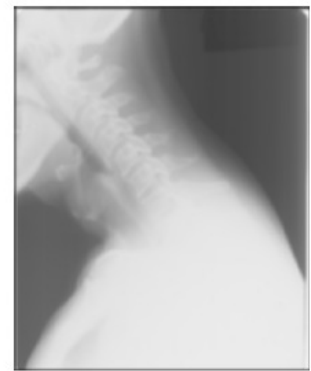

(b)

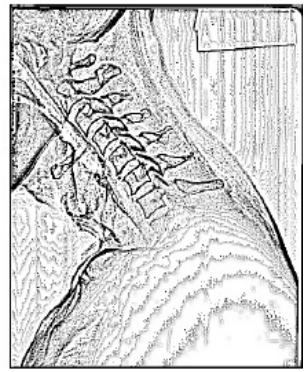

(c)

Figure 3. Unsharp masking (a) Original image (b) Gaussian smoothed image and (c) image after unsharp masking

It was found that the $3 \times 3$ optimum gradient operators yield the highest peak in the accumulator and the most compact and uniform region surrounding a possible candidate for the reference point.

\subsubsection{Definition of template:}

GHT is a template-based technique. It looks for instances of previously defined template in the target image. Even if good gradient information is available, the template must adequately represent the target object in order to obtain the necessary number of votes in the accumulator. The use of multiple templates should capture shape variability and closely represent the different classes of shapes seen across the data set. However, the use of a collection of templates may be computationally expensive. Consequently, the selection of a single template that represents the target object is of primary interest. As a first approximation, we have used the mean of 50 templates that were obtained from the manual land marking of 50 images [6] provided by the second National Health and Nutrition Examination survey (NHANES II) database [11]. The mathematical representation of the templates and that of the mean template is as follows:

$$
\begin{aligned}
& (\text { Template }) \mathrm{i}=[\mathrm{xi} 1, \ldots ., \mathrm{xi} 80, \mathrm{yi} 1, \ldots ., \mathrm{yi} 80]^{\wedge} \mathrm{T} \\
& \text { mean_template }=\frac{1}{50} \sum_{i=0}^{i=50}(\text { Template }) \mathrm{i}
\end{aligned}
$$

\subsubsection{Accumulator updating and reckoning of votes:}

Updating the accumulator, as well as picking the best estimate for the reference point is the remaining part of the algorithm.

\section{A. Hough Domain update process:}

Here, template matching is done based on the gradient information and for each match the accumulator bin is updated and this search and update process is repeated for Every value of scale's' \& rotation ' $\Phi$ '.A look-up table (R-table) for the template edge image is created with different values of $(\mathrm{x}, \mathrm{y})$, where $\mathrm{r}$ is the distance from the edge to the chosen reference point and $\alpha$ is the angle between the radius vector and the horizontal. The R-table summarizes the shape information of the template and is a part of the parametric representation of the image. Now, 
with the R-table, template matching is done on the target image and votes for possible matches are collected in the accumulator structure.

\section{B. Reckoning of votes in Hough Domain:}

The accumulator is a 4-D structure where 2 of the dimensions $(\mathrm{m} \times \mathrm{n})$ represent the spatial coordinates and the remaining two $(\mathrm{j} \times \mathrm{k})$ represent scale and rotation variations of the template. As a consequence, the 4-D accumulator can be seen as a ( $\mathrm{j} \mathrm{x} \mathrm{k)}$ collection of 2-D images of the spatial location of the reference and each of those images can be treated individually. If the 2-D accumulator is plotted for a fixed value of scale and rotation [Fig. 2(a)], we would expect as per theory, a well defined peak. In practice, the peak is neither unique nor well defined. Votes do not form uniform and compact regions and this makes the task of finding a good candidate for the reference point very difficult. Excessive noise in the original images makes votes sparse in the accumulator, yielding non-uniform structures. To fix this problem, Gaussian filtering is performed and this greatly improves the chances of finding an absolute maximum peak and this leads to a more relevant observation: Several peaks tend to align describing the orientation and location of the cervical vertebrae. The neighborhood peaks represent a more complete figure of merit about the overall performance of the template. A small window, having the highest peak as the central point is defined as our region of interest. The next step is to try and find the approximate orientation of the spine and the location of the neighboring peaks with the knowledge of the location of the highest peak. Let $\gamma$ be the angle that approximates the orientation of the template and ' $\Phi$ ' the current rotation angle of template. Then ' $\mu$ 'defined as

$$
\mu=\gamma+\Phi
$$

gives a good approximation of the orientation of the cervical spine in the target image. This angle is then used to rotate the small window as shown in Fig. 2(b). An intensity profile passing through the center of the window is defined as the characteristic profile and this has the property of passing through the center of the highest peak and if the rotation angle is correct, it should also pass through the neighboring peaks. A plot of the characteristic profile can be seen in Fig. 2(c). Once the highest peak has been found, the votes corresponding to this peak are eliminated, so that the next search yields the second highest peak. The same process is repeated and the third peak is also found. The output of the process will be $\hat{A}$, the cumulative value of the highest peak and its 2 neighbors. This step is a quantitative measure of how well the template matches the whole cervical vertebrae. The next subtlety involved in this analysis is the shape of the peaks. A blurred peak will indicate that in spite of the considerable quantity of votes that form the peak; the template matching is poor, yielding a widespread structure. On the other hand, a sharp peak will indicate a more accurate match since most of the votes concentrate in that small region. Hence, we use the gradient operator to measure the decay rate of the highest peak, and refer to this measure as $\hat{\mathrm{G}}$

The new criterion for reckoning of votes can be defined as a weighted sum of two quantities

$$
\text { Proposed criteria }=C(\hat{A})+\varepsilon(\hat{G})
$$

where $\beta \& \varepsilon$ are constant less than one.

For every value $(\Phi, s)$, a $2 \mathrm{D}$ image is created and for each of those we compute a pair of corresponding $(\hat{\mathrm{A}}, \hat{\mathrm{G}})$ values and store them. The $(\mathrm{x}, \mathrm{y})$ location of the highest peak is stored as the best candidate for the reference point. While the traditional criteria chooses the best $(\Phi, s)$ pair to be the highest value in the accumulator, the new criterion for reckoning of votes chooses the best $(\Phi, \mathrm{s})$ pair using both $\hat{\mathrm{A}}, \hat{\mathrm{G}}$ values as follows: 
1. Find the highest value in the accumulator for each value of scale and orientation.

2. Define the subset of each best $(\Phi, s)$ pair:

$$
(\Phi, \mathrm{s}) \text { best }=\{(\Phi, \mathrm{s}) \mid \hat{\mathrm{A}}(\Phi, \mathrm{s}) \geq \beta . \hat{\mathrm{A}}(\Phi, \mathrm{s})\}
$$

\subsection{VERTEBRAL FEATURES CALCULATION:}

In this section, four convex hull-based features are investigated for vertebra identification [2]. The following notation is used for defining the features. Let I denote the $\mathrm{M} \times \mathrm{N}$ intensity cervical spine X-ray image, where $1 \leq \mathrm{x} \leq \mathrm{M}$ and $1 \leq \mathrm{y} \leq \mathrm{N}$. Let $\mathrm{V}$ denote a cervical vertebra within an X-ray image with area Av such that

$$
\begin{array}{rlrl}
\mathrm{V} & =1 & \mathrm{if}(\mathrm{x} ; \mathrm{y}) \text { is on or inside the vertebra boundary } \\
& =0 & & \text { otherwise }
\end{array}
$$

The convex hull for $\mathrm{V}$ is determined using the quick convex hull algorithm [9]. Let $\mathrm{C}$ denote the resulting filled convex hull for vertebra $\mathrm{V}$ such that

$$
\begin{aligned}
\mathrm{C} & =1 \text { if }(\mathrm{x} ; \mathrm{y}) \text { is on or inside the convex hull boundary for } \\
& =0 \text { otherwise }
\end{aligned}
$$

Let $\mathrm{E}$ denote the set of exclusive-OR points between $\mathrm{V}$ and $\mathrm{C}$ such that

$\mathrm{E}(\mathrm{x}, \mathrm{y})=\mathrm{V}(\mathrm{x}, \mathrm{y}) \oplus \mathrm{C}(\mathrm{x}, \mathrm{y})$. $\mathrm{E}$ is expected to contain one or more connected regions, for each concave vertebral side. Using 8-connectivity, let $\mathrm{k}$ denote the number of unique connected components within E. The four features used for detecting anterior osteophytes include: (1) the ratio of the vertebral area to the convex hull area, (2) the ratio of the exclusive-OR area to the convex hull area, (3) the ratio of the exclusive-OR area on the vertebra's anterior side to the vertebra area and (4) the ratio of the area of the largest connected region from the exclusive-OR regions on the anterior side of the vertebra to the vertebra area.

The goal with feature extraction is to highlight protrusion regions on the anterior side of the vertebra that are characteristic of osteophytes. The ratios of the exclusive- OR area to the filled convex hull and the vertebra area, respectively, are intended to quantify the variation of the vertebral shape from the vertebra's ideal rectangular shape. The exclusive-OR regions represent concave regions around the vertebra's periphery. The final exclusive-OR-based feature focuses on the anterior side of the vertebra for finding the largest concave region and comparing the size of the concavity to the vertebra size in order to highlight the location of the largest protrusion region. The features examined provide size-invariant measures of the deviation of the filled convex hull from the original filled vertebra. The purpose for exploring size-invariant features is to enable the direct comparison of different vertebrae within the same X-ray image (i.e. the same patient) and vertebrae from different images (i.e. different patients). 


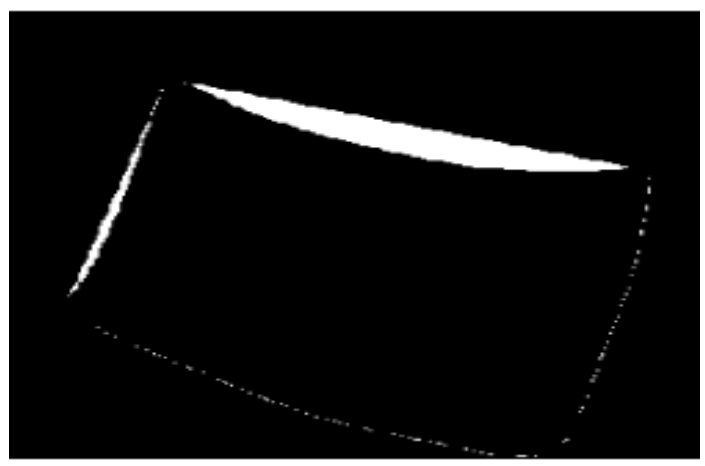

Figure (4): The exclusive-OR operation between original vertebra and its convex hull image for a normal vertebra.

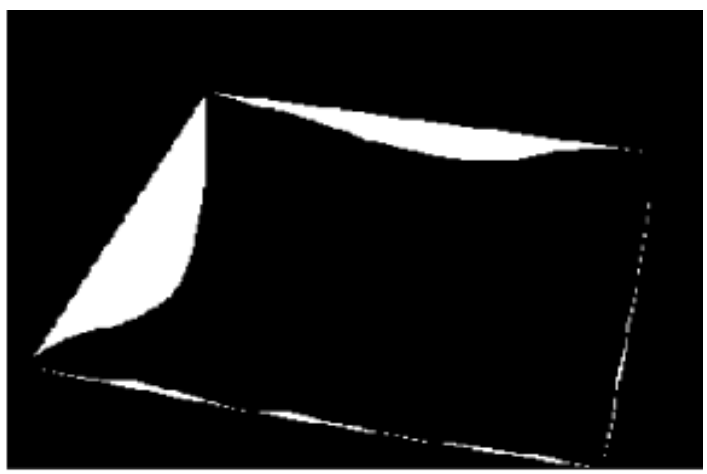

Figure (5). The exclusive-OR operation between original vertebra and its convex hull image for an abnormal vertebra with anterior osteophytes(lower left-hand side).

For the initial feature, the ratio of the vertebral area to the filled convex hull is denoted as $\mathrm{R}$ and is defined as $\mathrm{R}=\mathrm{AV} / \mathrm{AC}$ : The ratio of the exclusive-OR area to the filled convex hull area is denoted as $\mathrm{T}$ and is defined as $\mathrm{T}=\mathrm{AE} / \mathrm{AC}$ : In order to illustrate this feature, Figure(4) presents the exclusive-OR for a normal lumbar vertebra, and Figure(5) provides the exclusive-OR of an abnormal vertebra with anterior osteophytes on the lower left-hand side. From Figure (4) and (5), there are a number of disconnected regions in the exclusive-OR between the filled vertebra and its corresponding filled convex hull.

The following procedure is used to compute the final two exclusive-OR-based features. The centroid location, $(\mathrm{Xc}, \mathrm{Yc})$, is calculated for the vertebra V. Second, the connected regions within $\mathrm{E}$ are connected component labeled. Third, the original $\mathrm{XY}$ plane is shifted to new $\mathrm{X}^{1} \mathrm{Y}^{1}$ plane for $\mathrm{V}$, which is centered at the centroid location with the axes parallel to the original $\mathrm{XY}$ axes. 


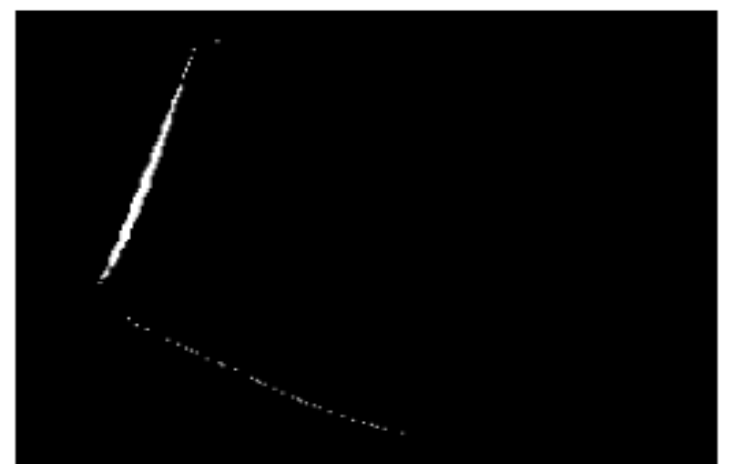

Figure(6). The exclusive-OR area after removing the posterior side of a normal vertebra. (Example for a normal vertebra).

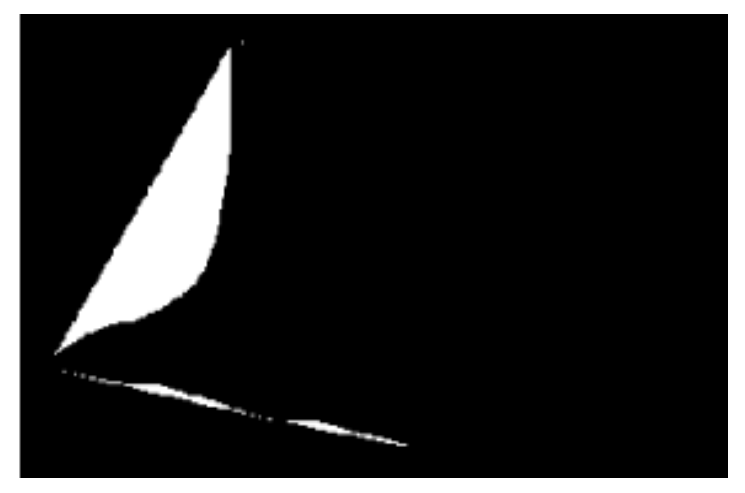

Figure (7). The exclusive-OR area after removing the posterior side of an abnormal vertebra

The third feature computed is the ratio of the exclusive-OR area on the vertebra's anterior side to the vertebral area. Connected components in $\mathrm{E}$ that touch or are entirely located on the righthand side of the $\mathrm{X}^{1} \mathrm{Y}^{1}$ plane (i.e. positive $\mathrm{X} 1$ half-plane) are removed. In other words, any connected component in $\mathrm{E}$ with one or more pixels on the $\mathrm{Y}^{1}$ axis or the negative $\mathrm{X}^{1}$ axis are removed. These connected components are considered as part of the posterior portion of the vertebra and are not related to anterior osteophytes. Let $\mathrm{G}$ denote the number of connected components remaining in the negative $X^{1}$ plane (i.e. anterior side). Let $H=\{h 1 h 2 \ldots h G\}$ refer to the set of connected components remaining with areas given by

$\mathrm{Ah}$; for $1 \leq \mathrm{i} \leq \mathrm{G}$ : Then, the ratio of the exclusive-OR area on the vertebra's anterior side to vertebral area is denoted as $U$ and is defined as $U=\sum_{i=1}^{G}$ Ahi / Av. The final convex hull-based feature is based on finding the largest connected component on the anterior side. Then, the ratio of the largest connected region from the exclusive-OR regions on the anterior side of the vertebra to the vertebral area is denoted as $\mathrm{F}$ and is defined as $\mathrm{F}=\operatorname{maxi}$ (Ahoi)/AV; where $1 \leq \mathrm{i} \leq \mathrm{G}$ : Note that $\mathrm{G} 1 / 41$ for the lumbar vertebrae examined. Figures 6 and 7 show examples of the exclusive-OR area after removing the posterior portion of the image for normal and abnormal vertebrae, respectively. 


\subsection{RELATED WORK:}

Abraham Tezmol, Hamed sari-sarraf , Sunanda Mitra, Rodney Long, Arunkumar gururajan perform customized hough transform for robust segmentation of cervical vertebrae from x-ray images.The aim of customized Hough transform (CHT), that captures shape variability and exploits shape information embedded in the accumulator structure to overcome noise and occlusions is proposed. This approach effectively finds estimates of the location and orientation of the cervical vertebrae boundaries in digitized $\mathrm{x}$-ray images.

Anterior osteophytes discrimination in lumbar vertebrae using size-invariant features, Image processing techniques are presented for computing size-invariant, convex hullbased features to highlight anterior osteophytes by Maruthi Cherukuria, R. Joe Stanleya, Rodney Longb, Sameer Antanib, George Thomab

In this proposed work Customized Hough transform will be used for segmentation \& size-invariant, convex hull-based features to locate anterior osteophytes in cervical vertebrae

\section{Conclusions}

In this research, image analysis techniques are presented for anterior osteophytes identification in cervical vertebrae. The task of segmenting the cervical vertebrae has been carried out using a customized Hough transform. This technique deals with shape information present in the voting structure. The gradient information \& template representation are very important aspects. For better shape variability more templates are used, approximately 50 templates give $100 \%$ shape variability. Four size-invariant, convex hull based features will be analyzed for differentiating normal vertebrae from abnormal vertebrae (containing anterior osteophytes). The convex hullbased features provide the variation in vertebra's shape from a typical convex shape, showing the variation on the anterior side of the vertebra. The ratio of the largest connected component on the anterior side from the exclusive-OR between the filled vertebra and its filled convex hull denoted as F. This will provide the highest and most consistent results of the four convex hullbased features.

Furthermore, the features investigated are size-invariant and can be applied to vertebra analysis in different X-ray images.

\section{REFERENCES}

[1] D.H. Ballard, "Generalizing the Hough Transform to detect arbitrary shapes", Pattern Recognition, 13(2), pp. 111-122, 1981.

[2] Maruthi Cherukuri "Anterior osteophyte discrimination in lumbar vertebrae using size-invariant features" Computerized Medical Imaging and Graphics 28 (2004) 99-108

[3] V.F. Leavers, "Which Hough Transform?”, Image Understanding, Vol. 58, No.2, pp. 250-264, Sep.1993.

[4] H. Li, M. A. Lavin, and R. L. LeMaster, "Fast Hough Transform”, Proceedings 3rd Workshop on ] Computer Vision, Bellair, MI, 1985. 
[5] J. Illingworth and J. Kittler, "The adaptive Hough Transform", IEEE Transactions on Pattern Analysis and Machine Intelligence, 9 (5), 1987.

[6] S. Ando, "Consistent gradient operators", IEEE Transactions on Pattern Analysis and Machine Intelligence, Vol. 22, No.3,

[7] Taylor JA, Resnick D. The aging spine: radiographic-pathologic correlation. In: Genant HK, Jergas M, van Juijk C, editors. Vertebral fracture in osteoporsis, research group. San Francisco, CA: University of California at San Francisco; 1995.

[8] Stanley RJ, Long R. A radius of curvature-based approach to cervical spine vertebra image analysis. Copper Mountain, CO. Proc 38th Annu Rocky Mountain Bioengng Symp 2001;37:385-90.

[9] Barber CB, Dobkin DP, Huhdanpaa HT. The Quickhull algorithm for convex hulls. ACM Trans Math Software 1996;22(4):469-83.Indian Institute of Science, Bangalore,India, Jan. 1999.

[10] AbrahamTezmol "Customized Hough Transform for Robust Segmentation of Cervical Vertebraefrom X-Ray Images" Fifth IEEE Southwest Symposium on Image Analysis and Interpretation (SSIAI.02) 0-7695-1537-1/02\$17.00@2002IEE

[11] Long LR, Thoma GR. Image query and indexing for digital X-rays.SPIE Conference on Storage and Retrieval for Image and Video Databases VII, San Jose, CA. Proc SPIE 1999;3656:12-21.

[12] TAYLOR JA, RESNiCK D. THE AGING SPINE: RADIOGRAPHIC-PATHOLOGIC CORRELATION. IN: GENANT HK, JERGAS M, VAN JUIJK C, EDITORS. VERTEBRAL FRACTURE IN OSTEOPORSIS, RESEARCH GROUP. SAN FRANCISCO, CA: UNIVERSITY OF CALIFORNIA AT SAN FRANCISCO; 1995.

[13] Stanley RJ, Long R. A radius of curvature-based approach to cervical spine vertebra image analysis. Copper Mountain, CO. Proc 38th AnnuRocky Mountain Bioengng Symp 2001;37:385-90.

[14] Genant HK, Ettinger B, Harris ST, Block JE, Steiger P. Quantitative computed tomography in assessment of osteoporosis. In: Riggs BL,Melton LJ, editors. Osteoporosis: etiology, diagnosis and management.New York: Lippincott Williams \& Wilkins Publishers.1988:221-49.

[15] Stanley RJ, Long R. A radius of curvature-based approach to cervical spine vertebra image analysis. Copper Mountain, CO. Proc 38th Annu Rocky Mountain Bioengng Symp 2001;37:385-90.

[16] Barber CB, Dobkin DP, Huhdanpaa HT. The Quickhull algorithm for convex hulls. ACM Trans Math Software 1996;22(4):469-83. 